\title{
SOBRE LA FORMA MUNCHO
}

\author{
ENRIQUE PATO \\ Université de Montréal \\ Enrique.pato-maldonado@umontrel.ca
}

\begin{abstract}
Resumen
La presente nota aporta luz nueva sobre la historia y la evolución de muncho en español. En primer lugar, los ejemplos presentados confirman que estamos ante un fenómeno antiguo, documentado en el mismo latín, y característico del español clásico. Estudiamos la variación que ha mantenido con la forma estándar mucho a lo largo de la historia y, gracias a los datos del ALPI y del COSER, damos a conocer su extensión geográfica. La $-n-$ parasitaria se documenta también en otras lenguas y dialectos romances, como el asturiano y los dialectos septentrionales italianos, así como históricamente en francés. Por último, revisamos el origen latino (muntum) y presentamos varios ejemplos de su extensión a otras palabras en español moderno.
\end{abstract}

PALABRAS CLAVE: Dialectología, Historia de la lengua, Español, Adverbio mucho, muncho.

\section{Introducción}

El adverbio muncho ha sido descrito como forma propia del judeoespañol (Subak, 1906: 173; Zamora Vicente, 1967: 361; Quintana Rodríguez, 2006: 217-220), del murciano (Zamora Vicente, 1967: 341; García Soriano, 1980; Díez de Revenga, 1997: 158; Gómez Ortín, 2004: 18; García Mouton, 2007: 35; Hernández Campoy, 2008), del andaluz (cf., entre otros, Cuervo, 1886: XLVII, n.1; Alcalá Venceslada, 1951: 421; Montes Giraldo, 1995-1996), del español de México y de Estados Unidos (Canfield, 1934: 75;

\footnotetext{
${ }^{1}$ Esta investigación se ha llevado a cabo gracias al proyecto KNOW2 (TIN2009-14715-C04) del Ministerio Español de Ciencia e Innovación.
}

This note provides new light on the history and evolution of Spanish adverb muncho. First, the examples presented confirm that this characteristic of Classical Spanish. We study the variation that has remained with of COSER data, we present its geographical extension. The parasitic $-n-$ is also documented in other Romance languages and dialects, as in Asturian, northern Italian dialects and, historically, in French. Finally, we review the Latin origin (muntum) and present several examples of its extension to other modern Spanish words.

KEY WORDS: Dialectology, History of Language, Spanish, Adverb mucho, muncho is an old phenomenon, documented in Latin, 
Henríquez Ureña, 1938: 374; Marden, 1938: 156; Company y Melis, 2002: 579-580; French, 2003; Marcos Marín, 2005: 335 y 2007: 135; Lipski, 2008: 94 y 218; Bills y Vigil, 2008: 69 y 166, etc.). Hoffman (1886: 301), por ejemplo, señaló "how corrupted the Spanish language has become in the south-west portion of the United States", citando entre otros el uso de muncho en lugar de mucho. Para Espinosa (1909: 26), en cambio, se trataría de "a popular form in all Spanish countries and frequent in Old Spanish", y así aparece en el DRAE (vid. muncho), como adjetivo 'anticuado y usado como vulgar', esto es común al español vulgar.

A simple vista, la forma muncho no presenta gran interés para la gramática histórica y dialectal, pero la aparición de esa nasal nos lleva a preguntarnos por varias cuestiones. Aparte de los trabajos clásicos de Luria (1937) y Cross (1938), ningún autor se ha preocupado ni por el origen ni por su documentación histórica y actual; desconocemos hasta cuándo se empleó de manera habitual en los textos, y si existe algún patrón geográfico-dialectal en su uso. Tampoco se ha estudiado la variación que ha mantenido con la forma estándar mucho a lo largo de la historia, ni se ha descrito la extensión de la epéntesis nasal a otras palabras. En el presente trabajo intentaremos dar respuesta a estas cuestiones pendientes.

\section{Breve descripción de la forma muncho}

Según el DRAE, muncho es un término propio de México (cf. las ediciones del DRAE de 1925, 1927, 1936, 1939, 1947 y 1950). Como "forma antigua y hoy vulgar", aparece desde 1956 (cf. las ediciones de 1956, 1970, 1984, 1989 y 1992).

Desde el punto de vista gramatical, al igual que mucho, muncho puede ser: 1) adjetivo, y su significado es el de 'abundante'. Como tal presenta variación de género y número (muncho, muncha, munchos, munchas); 2) cuantificador indefinido antepuesto al nombre (En Europa hay mu(n)cha crisis); 3) pronombre (Estuvo llamando a los restaurantes que había en la ciudad. No eran $m u(n)$ chos, la verdad); 4) adverbio (cuantificador de grado), con el significado de 'abundantemente o intensamente', aparece en ocasiones antepuesto a mejor, peor, mayor, menor, más, menos, antes y después; 5) presenta lectura inespecífica (o cardinal), entonces el SN que le precede tiene alcance amplio: No vinieron $m u(n)$ chos niños ('Hay muchos niños que no vinieron'); y 6) adquiere lectura colectiva y distributiva, con SSNN plurales y nombres no contables y coordinados; en este caso, la predicación se refiere a un grupo de entidades y no a los componentes individuales (NGLE, 2009), como en Sobre su escritorio se acumulaba mu(n)chos papeles, donde el valor distributivo se aplica a cada una de las entidades individuales que componen el conjunto. 


\section{Documentación de muncho}

Tal y como quedó indicado en la Introducción, es posible documentar la forma muncho (adverbio/ cuantificador > adjetivo y pronombre) desde el español medieval, sola (cf. 1a, c-d) o en variación con la forma mucho (cf. 1b). Los siguientes ejemplos ofrecen una breve muestra de ello:

(1)

a. diz essa estoria de las prouincias que uiene muncho Açafran \& muy bueno \& de meior olor que otro \& mas amariello que oro (Alfonso X, General estoria II).

b. Pues que les este castigo ouo dado bendixo los \& dixo. yd agora benditos \& con salut \& con muncho bien ca uos tornaredes a uuestros lugares loado a dios con muncho oro \& mucha plata \& mucho cobre \& muncho fierro \& munchos pannos de precio (Alfonso X, General estoria II).

c. De aquestas viejas todas ésta es la mejor; / ruégal' que te non mienta, muéstral' buen amor, / que muncha mala bestia vende buen corredor, / e muncha mala ropa cubre buen cobertor (Juan Ruiz, Libro de Buen Amor).

d. Bien cred que vna de las cosas en que los omnes muncho yerran es por que non saben beuir derecha mente con su muger et con sus fijos (Don Juan Manuel, Libro enfenido).

También es común en español clásico (cf. 2a), en judeoespañol (cf. 2b), en español coloquial (cf. 2c, ejemplo de México) y en hablantes bilingües (cf. 2d):

a. es el dicho Hernando de Aguilar hombre tan bullicioso, y escandaloso, e acostumbrado a tener munchas rebueltas con la justicia, como es notorio en esta cidad (Doc. 1491, AGS, Consejo Real, C. 611-9E, 30 de diciembre de 1557, Écija, Sevilla).

b. Kavamente la Sra. Sara Pardo, guida de Turismo de munchos anios publiko un libro remarkable en Turko sovre la Komunidad Djudia de Izmir, profiti muncho de esta dokumentasion. Kero mensionar kon grande apresiasion a la Sra. Rachel SabaWolfe de la kuala me embezi muncho sovre las luchas sosialas en el seno de la comunidad ("La komunidad djudia de Izmir (Smyrna)", Selim Amado, 2007).

c. Hola, me da muncho gusto que munchas mujeres creamos en la virgen, ya que munchas lamentablemente no creamos [sic], pero tengan muncha fe y créanme que se les cunpliran [sic] sus deseos, munchos saludos a todas y a todos, su amiga Elena ("Caminata de la Virgen de la Encarnación", T-amo-Dios, México, 13/05/2010).

d. cuando salió el conejo "ya me voy", dice "orita voy y vengo", dice "Te voy a trai un queso pa que te la coma", dice, "pa que no me vas a comer a mí", dice. Entons "bueno, ps ándale", dice "pero te viene pronto". "Sí, vengo pronto". Se jué el conejo y duró muncho y no podía venir... (Cruz del Palmar, Guanajuato, México, H, 75, bilingüe español-otomí). 


\section{Nuevos datos históricos}

Una de las primeras documentaciones de muncho ha sido presentada por SánchezPrieto (2008: 245), quien registró la fórmula ni poco ni muncho en un documento de 1236 (cf., además, Pascual y Blecua, 2006). Los datos del Corpus del español y del CORDE nos ayudan a fechar con mayor precisión el uso de muncho a lo largo de la historia de la lengua y a conocer el tipo de texto en que aparece. En concreto, y según los datos del CORDE (cf. Tabla 1 infra), es interesante comprobar que el $71 \%$ de todos los casos registrados de muncho son de 1509-1527 (primera mitad del s. XVI). En cuanto al tipo de texto, el 64\% figura en textos de prosa histórica. La diferencia que encontramos en la tabla 1 entre ambos corpus radica en que los textos que incluye el Corpus del español donde se registra muncho son casi todos de finales del siglo XV, como Cura de la piedra (1498) de J. Gutiérrez de Toledo, Valerio de las historias escolásticas y de España (1487) de D. Rodríguez de Almela, Crónica de España (1482) de D. de Valera, Tratado médico (o Tratado de la patología general), Libro del Cuzari de Jehuda ha-Levi y De los oficios, trad. A. de Cartagena. En el CORDE, el Tratado médico es considerado como texto del siglo XVI (1500), por ejemplo. Este corpus incluye otras obras de autores como Fray Bartolomé de las Casas o Gonzalo Fernández de Oviedo (prosa histórica) donde la documentación de muncho es constante 2 .

En resumen, como podemos comprobar en la tabla 1 de distribución de las formas muncho y mucho, la diferencia entre los dos corpus seleccionados (Corpus del español s. XV y CORDE s. XVI) viene marcada por la inclusión-exclusión de ciertos textos, pero los datos de ambos corpus confirman que las dos formas son empleadas principalmente en el siglo XVI, con porcentajes muy similares.

\begin{tabular}{llllllllll}
\hline \multirow{4}{*}{ Corpus del español } & & XIII & XIV & XV & XVI & XVII & XVIII & XIX & XX \\
& muncho & $5 \%$ & $0.3 \%$ & $\mathbf{8 4 \%}$ & $9 \%$ & $0.4 \%$ & $1 \%$ & $0.2 \%$ & -- \\
& & & & & \\
CORDE & muncho & $3 \%$ & $4 \%$ & $21 \%$ & $\mathbf{7 1 \%}$ & $1 \%$ & --- & --- & --- \\
& mucho & $3 \%$ & $5 \%$ & $13 \%$ & $\mathbf{2 9} \%$ & $19 \%$ & $6 \%$ & $11 \%$ & $14 \%$ \\
\hline
\end{tabular}

Tabla 1. Distribución de muncho y mucho, por tipo de corpus, a lo largo de la historia de la lengua.

\footnotetext{
${ }^{2}$ Para una revisión más detallada de los textos y autores en donde es posible la documentación de la forma muncho, y los problemas de edición que conlleva (mũcho> muncho), cf. el trabajo de Pascual y Blecua (2006: 1363-1370).
} 
Por otro lado y como cabría esperar, muncho muestra un comportamiento similar al de mucho en cuanto a la documentación de sus formas (variación de género y número). En efecto, tanto la documentación de mucho (246.400 casos en el Corpus del español y 554.459 en el CORDE) como la de muncho (3.185 en el Corpus del español y 8.834 en el CORDE) ofrecen porcentajes de aparición que favorecen la forma del masculino singular, seguido de las formas de plural (cf. Pato, 2011).

\begin{tabular}{lrllllll}
\hline \multicolumn{3}{c}{ Corpus del español } & \multicolumn{4}{c}{ CORDE } \\
mucho & $\mathbf{4 1} \%$ & muncho & $\mathbf{4 0 \%}$ & mucho & $\mathbf{3 8} \%$ & muncho & $\mathbf{3 4} \%$ \\
mucha & $10 \%$ & muncha & $13 \%$ & mucha & $12 \%$ & muncha & $12 \%$ \\
muchos & $26 \%$ & munchos & $20 \%$ & muchos & $27 \%$ & munchos & $25 \%$ \\
muchas & $23 \%$ & munchas & $27 \%$ & muchas & $23 \%$ & munchas & $29 \%$ \\
\hline
\end{tabular}

Tabla 2. Distribución de mucho y muncho (variación de género y número), por tipo de corpus.

Como vemos en la tabla 2, en porcentajes totales mucho alcanza un $98.52 \%$ (800.859 casos) y muncho un $1.47 \%$ (12.019 casos). Datos que se asemejan a lo encontrado en el español de México por Company y Melis (2002: 579-580): mucho 95\% (692 casos) vs. muncho 5\% (38 casos).

En otros corpus lingüísticos, como el de Biblia Medieval, la forma muncho se documenta exclusivamente en las versiones romanceadas traducidas del canon hebreo de la primera mitad del siglo XV (71 casos). En efecto, se registra en la Biblia de Ajuda (14201430), el códice escurialense I.i.5 (1420-1445), la Biblia de Évora (1429, traducción que coincide con E5), el ms. 10.288 de la Biblioteca Nacional de Madrid (primera mitad del siglo XV), el ms. 87 de la Biblioteca de la Real Academia de la Historia (1450-1475) y la Biblia de Alba (1422-1430). Esta situación puede deberse a un condicionamiento cronológico, y no a la lengua subyacente del modelo o a la variedad romance de los traductores, ya que no aparece ni en la Fazienda ni en los manuscritos E6/E8 del siglo XIII. Aparece preferentemente como adverbio de cantidad, pero también como adjetivo.

Por último, en los vocabularios del XVI-XVII como el Vocabulario de las dos lenguas toscana y castellana de Cristóbal de las Casas (1576: 240) troppo se traduce como 'muncho', y en el Tesoro de las tres lenguas, española, francesa e italiana de Vittori (1644: 168) mont es el equivalente de 'moult, molto, mucho, muncho'.

\section{Extensión geográfica, histórica y actual}

Desde el punto de vista histórico, los datos del CODEA confirman la documentación de la forma muncho desde 1496 a 1625, especialmente en textos notariales de Écija (Sevilla), Granada y Calatayud. También aparece, aunque en menor proporción, en 
documentos notariales de Burgos, Toledo y Madrid. Los sustantivos a los que cuantifica son gente, personas, armas, rebueltas, beneficio y donaciones, aunque también figuran sustantivos coordinados del tipo escándalo e alboroto, daño y perjuizio.

Los datos que guarda el ALPI, datos ya histórico-dialectales, arrojan luz nueva sobre el fenómeno en estudio. Aunque algunas de las preguntas del cuestionario gramatical no fueron completadas en varios de los cuadernos, gracias a las preguntas Mucho (Cuaderno I, p. $8 \# 76)^{3}$ y Mucha cosecha (Cuaderno I, p. $\left.4 \# c h\right)^{4}$ podemos obtener una imagen precisa de su extensión geográfica en la península antes de la guerra civil; imagen que permite corroborar en parte la distribución de los datos de las descripciones previas.

El siguiente mapa, presentado por primera vez, muestra la extensión de muncho (y variantes) en el ALPI. Como podemos comprobar, la forma vernácula se registra en el centro y este de Asturias ${ }^{5}$, de manera esporádica en Aragón (sur de Zaragoza y Teruel) ${ }^{6}$ y Murcia ${ }^{7}$ y de manera general en Andalucía, en las provincias de Cádiz, Sevilla, Córdoba, Málaga, Granada y Jaén, así como en el sur de Ciudad Real.

\footnotetext{
${ }^{3} \mathrm{La}$ forma munchu se registra en los enclaves $311,312,313,315,316,317,318,319,320$, 321. La forma muntcho en 503, 505, 507, 508, 509, 512, 513, 514, 516. Los casos de muncho/ munsho en 314, 332, 480, $481,504,506,510,511,515,524,527,528,529,530,531,532,534,535,536,537,538,539,540,541$, $542,543,544,545,546,547,548,549,550,551,552,566,625,635,636,638$. Y por último muntu en 365.

${ }^{4}$ Las formas muncha (y muntcha) aparecen documentadas en los puntos de encuesta $315,320,321$, $332,480,481,625,635,638,504,505,506,507,508,509,510,511,512,513,514,515,516,524,527$, $529,530,532,534,535,536,537,538,539,540,541,542,543,544,545,546,547,548,549,550,551$, 552, 566. La forma munta en 365.

${ }^{5} \mathrm{La}$-n- epentética es habitual en asturiano (cf. García Arias, 2003: 250). Como es sabido, forma el adverbio de cantidad: trabaya muncho bien; son muncho bones (cf. ALlA, 2002: 45; ALlA, 2008: 156).

${ }^{6}$ Khun (2008: 111) registró en los años 30 casos 'espontáneos' de epéntesis consonántica en altoaragonés (Ansó, Hecho y Sallent), como henchizar ('hechizar') y henchicero ('hechicero').

${ }^{7}$ En este caso en particular, los datos del ALPI resultan un poco limitados, ya que la forma muncho constituye uno de los rasgos caracterizadores del murciano (cf., entre otros, Zamora Vicente, 1967: 341; García Mouton, 2007: 35; y desde el punto de vista histórico, Díez de Revenga, 1997: 158).
} 


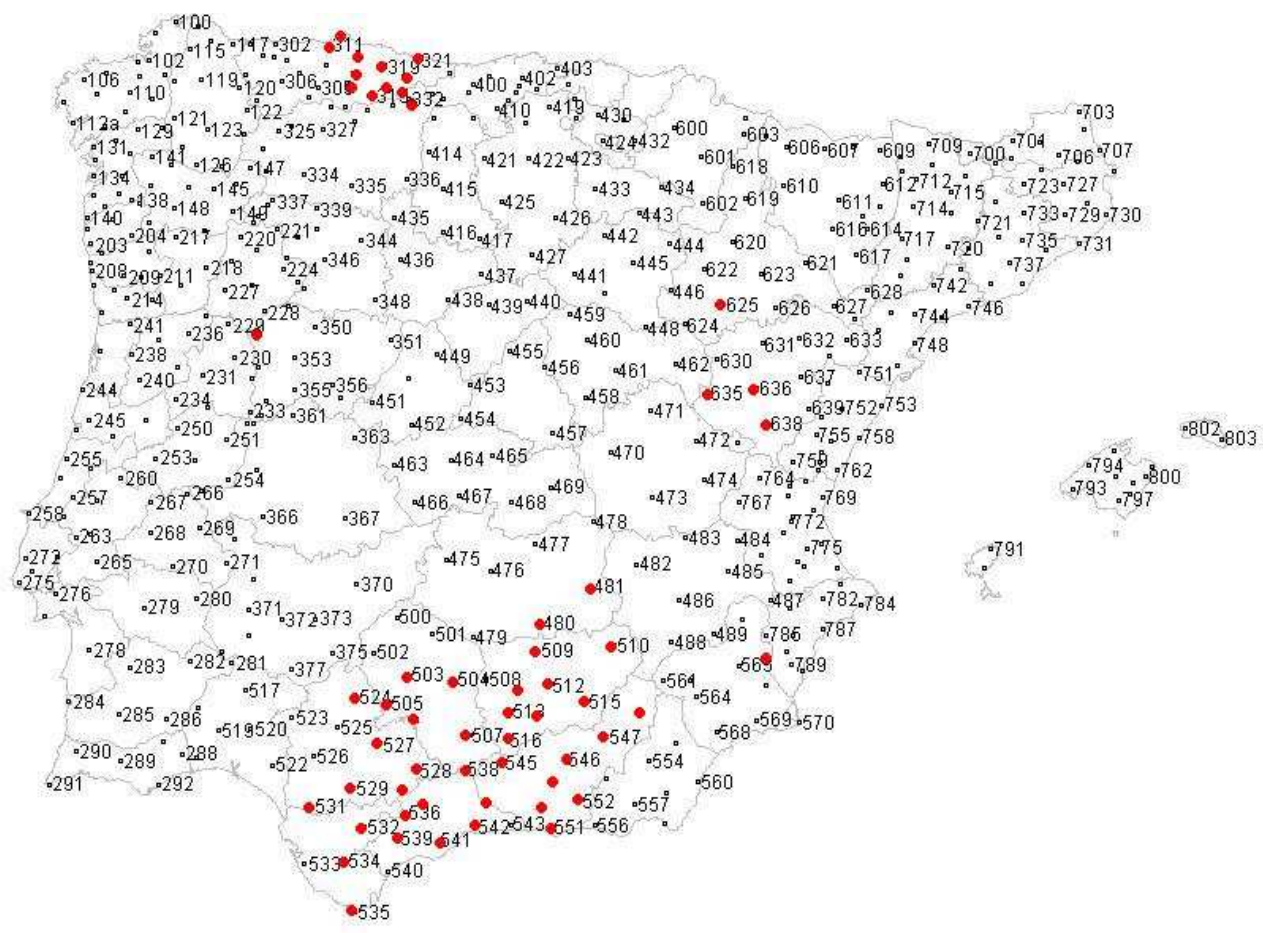

Mapa 1. Extensión de la forma muncho (y variantes), según los datos del ALPI.

Por último, los datos del COSER confirman, también, su uso pleno en Andalucía. La documentación sistemática de la forma muncho (en casi total ausencia de la estándar mucho) hace pensar en un proceso antiguo de lexicalización de un fenómeno fonético, que ha conducido a muncho a ser la forma aprendida y transmitida en el español rural de Andalucía, del mismo modo que ha ocurrido con otras palabras, especialmente adverbios, que en su proceso de fijación han añadido la nasal, tal es el caso de asín y ansí ('asi', cf. Rodríguez Molina, 2012), lenjos ('lejos', derivado de longe, cf. Zamora Vicente, 1967: 341) o mejón ('mejor', de origen andaluz, cf. Casares, 1992: 275, n. 1), entre otros.

\footnotetext{
${ }^{8}$ En su Diálogo de la lengua, Valdés hace referencia a este adverbio en estos términos: "MARCIO: Dos vocablos hallo de los quales vos, no sé por qué, quitáis una n, diziendo por invierno y lenxos, ivierno y lexos. ¿Hazéislo por industria o por descuido? VALDÉS: El descuido creo yo que está en los que ponen la $\mathrm{n}$ sin propósito ninguno. $\mathrm{Y}$ ésta es una de las letras que yo digo que por inadvertenzia se an mezclado en algunos vocablos".
} 
Los siguientes ejemplos, fruto de la campaña de encuesta realizada en septiembre de 2012, son solo una breve muestra de la rica documentación que guarda el COSER sobre el fenómeno:

(3)

a. A mí no, a mí me gusta que esté más, más cuajá [la morcilla]. Sí, pero... estaba estupenda. Sí. Yo he hecho muncha (Cañete de las Torres, Córdoba, COSER-1517).

b. ¿Con el trigo?, lo vendían; aquí había una fábrica de harina, lo cogían mayormente y se lo llevaban pa' fuera, ahí a los, a las comarcales, que decían "comarcal", y esas cosas, y na' luego lo cogían muncho, lo cogían y lo llevaban a una fábrica ahí pa' molerlo, pa' hacer pan (Sierra de Yeguas, Málaga, COSER-3015).

c. Eso estaba todo, cuando Marbella empezó... estaba todo de hoteles, y todo el mundo se fue allí. ¡Las muchachas ganaban muncho dinero! Mucho dinero han ganao... (Jubrique, Málaga, COSER-3009).

\section{Posible origen y fuente de analogía}

Según Menéndez Pidal (1968), la adición de la nasal podría explicarse por 'reflejo' de otra nasal que hay en la misma palabra ( $\underline{m} u c h o>$ muncho). Grandgent (cit. en Cross, 1938: 601) señaló el paso multu> muntu en el mismo latín (forma documentada también en el ALPI, si bien de manera esporádica, cf. las notas 3 y 4), y otros autores como Sommer (1914: 168) y Stolz-Schmalz (1928: 167) incluyeron el estudio de estas formas en sus trabajos. Para todos ellos la fuente de analogía (en latín) podría estar en los adjetivos de cantidad: tantum/ quantum/ multum $>$ muntum. De hecho, es posible documentar la adición de la - $n$ - en el Corpus Inscriptionum Latinarum (1863-1864: 1593) «SI VALES NON MVNTV CVRO», en el Appendix Probi «cultellum non cuntellum», así como en las pizarras visigodas «nontas» por notas (cf. Velázquez Soriano, 2004: 194).

Desde el punto de vista románico y tipológico, la nasalización del grupo -lt-> -nt(molto $>$ monto) se registra en algunos dialectos italianos (Parma y Génova, por ejemplo): monto bene, monto dificile; y en francés la $-n$ - 'parasita' ha aparecido en varias palabras ante consonante oclusiva, como langouste (LOCUSTA), concombre (fr.a. cocombre), jongleur (fr.a. jougleur 'malabarista'); o nasal, como tampon (fr.a. tapon), etc. (cf., entre otros, Nyrop, 1899: 375).

\footnotetext{
${ }^{9}$ Agradezco a Inés Fernández-Ordóñez (UAM/RAE) la consulta de los materiales del COSER, y a Carlota de Benito (UAM) la ayuda que me ofreció para localizar los ejemplos. La primera transcripción de los datos es mía.
} 
Por otro lado, el grupo - nch- ha sido muy productivo en español. Baste citar los casos de ancho (AMPLUS), concha (CONCHULA) o cincha (CINGULA), numerosos préstamos como lancha (port.), plancha (fr.) o poncho (¿quechua, mapudungun?), así como voces de origen incierto como gancho, pinche, etc. Además, aparece en palabras que etimológicamente no presentaban esa $-n$ - (ante consonante oclusiva de nuevo), como mancha (MACULA), mancilla (lat.vulg. MACELLA), ninguno ( $<$ NEC UNUS), manzana (mazana <MATTIANA), o en préstamos, como mensaje (prov. messatge). Tal y como han mostrado varios autores (cf., por ejemplo, Pharies 1994: 4), la nasal se registra a su vez en palabras derivadas como flaco $>$ flacuncho (Alvar, 1972: 31) o gordo> gordoncho (Alcalá Venceslada, 1951: 302). Aparece incluso con aumentativos (munchísimo, cf. 4a) y ha creado desde antiguo derivados del tipo munchedumbre ('muchedumbre', cf. 4b-c):

(4)

a. Hacía munchos, munchísimos años que en nuestro pueblo no era menester castigar a ningún malnacido por una falta como la tuya (Ramón Rubín, El canto de la grilla, 1952, México).

b. Et sobresto cuenta Josepho que Josue \& los de Jsrahel quando sopieron que tamanna era la munchedunbre destas yentes \& del guisamiento que trayen que dubdaron de yr a ellos (Alfonso X, General estoria II).

c. A los del linage de Joseph fazien se les poco las suertes delos heredamientos que auien. Et mostraron lo alli a Josue \& dixieron le assi / Por que nos diste por heredat tierra duna suerte \& duna medida; seyendo nos tamanna muchedunbre cuemo somos \& nos bendixo nuestro sennor \& nos acrescio assi. bendito sea ell (Alfonso X, General estoria II).

La extensión de la - $n$ - a otras palabras no ha recibido, que sepamos, atención por parte de ningún autor. La analogía con el grupo -nch- (concha, plancha, etc.) ha creado palabras como mancho ('macho', Sos muy mancho, chavón); manchete ('machete', Agarró a mi gata doméstica y le cortó el cuello con un manchete); mencha ('mecha'), menchero ('mechero'), menchones ('mechones'), monchila ('mochila'), munchachos ('muchachos'), cunchillo ('cuchillo'), chinchón ('chichón'), oncho ('ocho'), aprovenchar ('aprovechar'), anonchecer ('anochecer'), lunchar ('luchar'), henchizar ('hechizar'), nincho ('nicho'), etc. ${ }^{10}, y$ así se pueden registrar en varios corpus lingüísticos y en diversas fuentes en línea (cf. Pato, 2011). Aparece incluso con nombres propios, Manchín ('Machín',

\footnotetext{
${ }^{10} \mathrm{La}$-n- epentética se registra también en algunos enclaves de Alicante, como Villena, bolinche ('boliche'), calinche ('caliche'), moncho ('mocho'), etc., y en valenciano (ninxo, enxisar). Cf. Torreblanca (1976: 165) y Domene Verdú (2010: 227).
} 
Escucha las canciones de Antonio Manchín), Nancho ('Nacho'), y se extiende también al interior de palabras que ya presentan el grupo -nch-, como en zanfarrancho ('zafarrancho') (cf. Rodríguez Izquierdo, 1994: 46).

\section{Consideraciones finales}

Si bien Meyer-Lübke (1890: 519) adelantó una explicación sobre la asimilación nasal progresiva, por el que la $/ \mathrm{n} /$ nasaliza el comienzo plosivo del elemento africado (ch), la manera en que se produce la transmisión de los rasgos fónicos ha sido muy poco estudiada. Un trabajo fonológico sobre la adquisición de la lengua materna, y la lengua segunda, podría dar cuenta de este fenómeno.

Un trabajo piloto llevado a cabo en la Universidad de Montreal (cf. Pato, 2011) con 20 informantes (5 estudiantes nativos, 13 estudiantes francófonos de ELE y 2 personas sin competencia alguna en español) nos ha mostrado que la aparición de la nasal /n/ ayuda a los informantes en la pronunciación de la -ch-(hacha/ ancha; poncho/ pocho). Como es sabido, la pronunciación de las africadas exige una fase de obstrucción total y otra de fricción. En la primera etapa, el flujo del aire se interrumpe, y en la segunda, escapa forzadamente; es decir, el predorso de la lengua se separa gradualmente, creando una estrechez por donde escapa el aire produciendo así la fricación. Por tanto, y de forma natural, cuando se pronuncia la ch cuesta forzar el aire a que salga por la boca después de la fricción. En la pronunciación del grupo -nch-, en cambio, la lengua se eleva para hacer contacto con el paladar duro, y el predorso de la lengua se adhiere al prepaladar y los alvéolos, impidiendo la salida del aire por la boca, lo que facilita su pronunciación. Con todo, es posible considerar la aparición de la nasal (muncho vs. mucho) como elemento que ayuda en la emisión de la $\mathrm{ch}$, al menos para los informantes no nativos (90\% en alumnos de ELE y $100 \%$ en personas sin competencia en español) ${ }^{11}$.

La elaboración del presente trabajo nos ha permitido obtener una serie de resultados, hasta ahora desconocidos sobre el fenómeno en estudio, que aportan luz nueva sobre la historia y la evolución de muncho en español. En primer lugar, como hemos visto en los ejemplos presentados, estamos ante un fenómeno antiguo, documentado en el mismo latín, y característico del español clásico, en concreto del primer cuarto del siglo XVI (textos fechados entre 1509 y 1527). La -n- 'adventicia' se documenta tam-

\footnotetext{
${ }^{11}$ Habría que comprobar ahora en los datos del COSER si, allí donde la fricativización de la -ch- es más frecuente, como por ejemplo en las provincias de Sevilla y Cádiz (musho), los casos de -n(munsho) presentan un porcentaje de aparición menor.
} 
bién en otras lenguas y dialectos romances, como el asturiano y los dialectos septentrionales italianos, así como históricamente en francés. Gracias a los datos del ALPI y del COSER hemos conocido la extensión geográfica de muncho, que lo presentan como rasgo característico del andaluz. Por último, hemos revisado el posible origen (lat. muntum) y presentado varios ejemplos de la difusión de la $-n$ - a otras palabras en español moderno.

Recibido: 24-X-2012

Aceptado: 02-IV-2013

\section{Referencias bibliográficas}

Academia de la Llingua Asturiana. (2002): Informe sobre la Llingua Asturiana. Oviedo, Academia de la Llingua Asturiana.

Academia de la Llingua Asturiana. (2008): Gramática de la Llingua Asturiana. Oviedo, Academia de la Llingua Asturiana.

Alcalá Venceslada, A. (1951): Vocabulario andaluz. Madrid, Real Academia Española.

ALPI. Atlas Lingüistico de la Península Ibérica (en línea), Heap, D. (coord.), The University of Western Ontario: http://www.alpi.ca (20-04-2011).

Alvar, M. (1972): Cuestionario para el estudio coordinado de la norma lingüistica culta de las principales ciudades de Iberoamérica y de la Península Ibérica. Vol. 2: Morfosintaxis. Madrid, CSIC.

Biblia Medieval, Enrique-Arias, A. (dir.), Universidad de las Islas Baleares: http://www.bibliamedieval.com (20-04-2012).

Bills, G. D. y N. A. Vigil. (2008): The Spanish Language of New Mexico and Southern Colorado. A Linguistic Atlas. Nuevo México, The University of New Mexico Press.

Canfield, D. L. (1934): Spanish literature in Mexican languages as a source for the study of Spanish pronunciation. Nueva York, Instituto de las Españas en Estados Unidos.

Casares, J. (1992): Introducción a la lexicografía moderna. Madrid, CSIC.

CODEA. Corpus de Documentos Españoles anteriores a 1700. Universidad de Alcalá: http://demos.bitext.com/codea/codea_form.asp (22-03-2012).

Company, C. y C. Melis. (2002): Léxico histórico del español de México. Régimen, clases funcionales, usos sintácticos, frecuencias y variación gráfica. México, UNAM.

CORDE. Corpus diacrónico del español. Madrid, Real Academia Española: http://www.rae.es (28-10-2012).

Corpus del español, Davies, M. (dir.), Brigham Young University: http://www.corpusdelespanol.org (28-10-2012).

COSER. Corpus Oral y Sonoro del Español Rural, Fernández-Ordóñez, I. (dir.), Univer- 
sidad Autónoma de Madrid: http://www.coser.uam (28-10-2012).

CREA. Corpus de referencia del español actual. Madrid, Real Academia Española, www.rae.es (28-10-2012).

Cross, E. (1938): "On Spanish muncho, Portuguese muito", Modern Language Notes, $53 / 8$, págs. 600-602.

Cuervo, R. J. (1886): Diccionario de construcción y régimen de la lengua castellana. París, A Roger y F. Chernoviz.

De las Casas, C. (1576): Vocabulario de las dos lenguas toscana y castellana. Sevilla, Francisco de Aguilar.

Díez de Revenga Torres, P. (1997): "Observaciones filológicas sobre aspectos relativos al medio ambiente en la documentación notarial”, Epos, XIII, págs. 151-170.

Domene Verdú, J. F. (2010): "Caracterización lingüística del habla de Villena (Alicante)”, Archivo de Filología Aragonesa, 66, págs. 217-243.

Espinosa, A. M. (1909): Studies in New Mexican Spanish. Part I: Phonology. Chicago, The Chicago University Press.

French, W. E. (2003): “Te Amo Muncho: The Love Letters of Pedro and Enriqueta". En Pilcher, F. M. (ed.): The Human Tradition in Mexico. Wilmington (DE), Scholarly Resources, págs. 123-135.

García Arias, X. L. (2003): Gramática histórica de la lengua asturiana. Oviedo, Academia de la Llingua Asturiana.

García Mouton, P. (2007): Lenguas y dialectos de España. Madrid, Arco/ Libros.

García Soriano, J. (1980): Vocabulario del dialecto murciano. Murcia, Editoria Regional.

Gómez Ortín, F. (2004): "El dialecto murciano y sus variedades", Tonos digital, 8, págs. 7-27.

Henríquez Ureña, P. (1938): "Mutaciones articulatorias en el habla popular". En Henríquez Ureña, P. et alii. (eds.): El español en Méjico, los Estados Unidos y la América Central. Buenos Aires, Imprenta de la Universidad de Buenos Aires, págs. 329-379.

Hernández-Campoy, J. M. (2008): “Sociolinguistic aspects of Murcian Spanish”, International Journal of the Sociology of Language, 193/194, págs. 121-138.

Hoffman, W. J. (1886): "Remarks on Indian Tribal Names". En Proceedings of the American Philosophical Society. Philadelphia, M'Calla \& Stavely, págs. 294-303.

Khun, A. (2008): El Dialecto Altoaragonés. Zaragoza, Xordica-Prensas Universitarias de Zaragoza.

Lispki, J. M. (2008): Varieties of Spanish in the United States. Washington, Georgetown University Press.

Luria, M. A. (1937): "Old and Dialectical Spanish muncho, Portuguese muito", Language, 13/4, págs. 317-318. 
Marcos Marín, F. (2005): "Pluralidad del español en los Estados Unidos de América". En El Español en el Mundo. Anuario del Instituto Cervantes 2005. Barcelona, Plaza \& Janés/ Círculo de Lectores, págs, 283-357.

Marcos Marín, F. (2007): "Tres líneas léxicas del español en los Estados Unidos de América". En Actas del I Congreso Internacional de Léxico Español Actual. Venecia, Librería Editrice Cafoscarina, págs. 127-141.

Marden, C. C. (1938): "La fonología del español en la ciudad de Méjico". En Henríquez Ureña, P. et alii. (eds.): El español en Méjico, los Estados Unidos y la América Central. Buenos Aires, Imprenta de la Universidad de Buenos Aires, págs. 87-187.

Menéndez Pidal, R. (1968): Manual de Gramática Histórica Española. Madrid, EspasaCalpe.

Meyer-Lübke, W. (1890): Grammatik der romanischen Sprachen. Leipzig, Fues's Verlag.

Montes Giraldo, J. J. (1995-1996): "La bipartición dialectal del español”, Boletín de Filología de la Universidad de Chile, 35, págs. 317-331.

Nyrop, K. (1899): Grammaire historique de la langue française. París, A. Picard \& fills.

Pascual, J. A. y J. M. Blecua. (2006): "De los munchos tipos de $n$ adventicia del español". En Filología y Lingüística. Estudios ofrecidos a Antonio Quilis. Madrid, CSIC/UNED, vol. II, págs. 1361-1384.

Pato, E. (2011): "La historia de mucho/ muncho en español", ponencia presentada en el 64th Annual Kentucky Foreign Language Conference. Lexington, University of Kentucky (14-16 de abril de 2011).

Pharies, D. (1994): “Origin of the Spanish -nch- Suffixes”, Iberoromania, 40, págs. 1-43.

Quintana Rodríguez, A. (2006): Geografía lingüística del judeoespañol. Estudio sincrónico y diacrónico. Bern, Peter Lang.

Real Academia Española. (2009): Nueva gramática de la lengua española. Madrid, Espasa/ Real Academia Española.

Rodríguez Izquierdo, F. (1994): "Voces con eco nasal", $A B C$ (Sevilla), 5 de diciembre de 1994, pág. 46.

Rodríguez Molina, J. (2012): “El adverbio así en español medieval: variantes morfológicas", ponencia presentada en el IX Congreso Internacional de Historia de la Lengua Española. Cádiz, Universidad de Cádiz (10-14 de septiembre de 2012).

Sánchez-Prieto Borja, P. (2008): "La variación lingüística en los documentos de la catedral de Toledo". En Elvira, J. et alii. (eds.): Lenguas, reinos y dialectos en la Edad Media Ibérica: la construcción de la identidad. Homenaje a Juan Ramón Lodares. Madrid/ Frankfurt, Iberoamericana/ Vervuert, págs, 233-256.

Sommer, F. (1914): Handbuch der lateinischen Laut- und Formenlehre. Heidelberg, C. Winter.

Stolz, F. y J. H. Schmalz. (1928): Lateinische Grammatik. Laut-und Formenlehre, Syntax 
und Stilistik. Munich, C. H. Beck.

Subak, J. (1906): “Zum Jüdenspanischen”, Zeitschrift für Romanische Philologie, XXX, págs. 129-185.

Torreblanca, M. (1976): Estudio del habla de Villena y su comarca. Alicante, Instituto de Estudios Alicantinos.

Velázquez Soriano, I. (2004): Las pizarras visigodas. Burgos, Instituto Castellano y Leonés de la Lengua/ Real Academia Española.

Vittori, G. (1644): Tesoro de las tres lenguas, española, francesa e italiana. Ginebra, Jaques Crespin.

Zamora Vicente, A. (1967): Dialectología española. Madrid, Gredos. 